\title{
Tracing Based Segmentation for the Labeling of Individual Rib Structures in Chest CT Volume Data
}

\author{
Hong Shen ${ }^{1}$, Lichen Liang ${ }^{2}$, Min Shao ${ }^{3}$, and Shuping Qing ${ }^{1}$ \\ ${ }^{1}$ Siemens Corporate Research, Inc., 755 College Road East, Princeton, NJ 08540, USA, \\ \{shenh, sqing\}@scr.siemens.com \\ ${ }^{2}$ Electrical and Computer Engineering Department, University of Minnesota, Minneapolis, \\ MN 55455, USA, lianglc@ece.umn. edu \\ ${ }^{3}$ Department of Electrical and Computer Engineering, University of Delaware, Newark, DE \\ 19716, USA, 55298@udel. edu
}

\begin{abstract}
We propose a fast and robust segmentation algorithm for the extraction and labeling of individual rib structures in chest CT volume data. A diagnostic system based on this algorithm can display 3D rib centerlines, contours and surfaces. A click on a rib point in any slice image will have the system identify instantly the individual rib it belongs to. The algorithm is based on a recursive tracing approach. The geometrical properties of the rib structure are explored to set up valid assumptions and model. At each step, statistical analysis is combined with dynamic programming to estimate the outer surface contour from the detected edges. The algorithm works reliably on CT volume data of variant doses and resolutions. This algorithm can be extended to other modalities. The detected centerlines can also be used for reliable and fast registration of in or cross-modality volume data of chest scans.
\end{abstract}

\section{Introduction}

The advances in medical imaging equipment have brought efficiency and high capability to the screening, diagnosis and surgery of various kinds of diseases. The $3 \mathrm{~d}$ imaging modalities, such as multi-slice CT scanners, produce large amount of digital data that is difficult and tedious to be interpreted merely by physicians. Computer Aided Diagnosis (CAD) systems will therefore play a critical role, especially in the visualization, segmentation, detection, registration, and reporting of medical pathologies.[1][2] Due to the large volume of the data involved and the on-time requirement of medical applications, feature based algorithms will be one of the most frequently applied methods to accomplish these tasks effectively and efficiently.

The extraction of the rib structure has significant meanings to achieve several of the above functionalities to be provided by a CAD system. There are 12 pairs of rib structures in the human body, with 8-10 pairs visible in a chest CT volume data. They are connected at one end with the spine, and the upper ribs are also connected to the sternum. As an anatomy, the visualization and labeling of rib structures are of great interest to radiologist, since they need to report any pathology pertaining to bones in a 
chest CT scan. A mouse click on a rib in the volume data should have the system instantly identify the rib. This function is highly desirable by a chest radiologist.
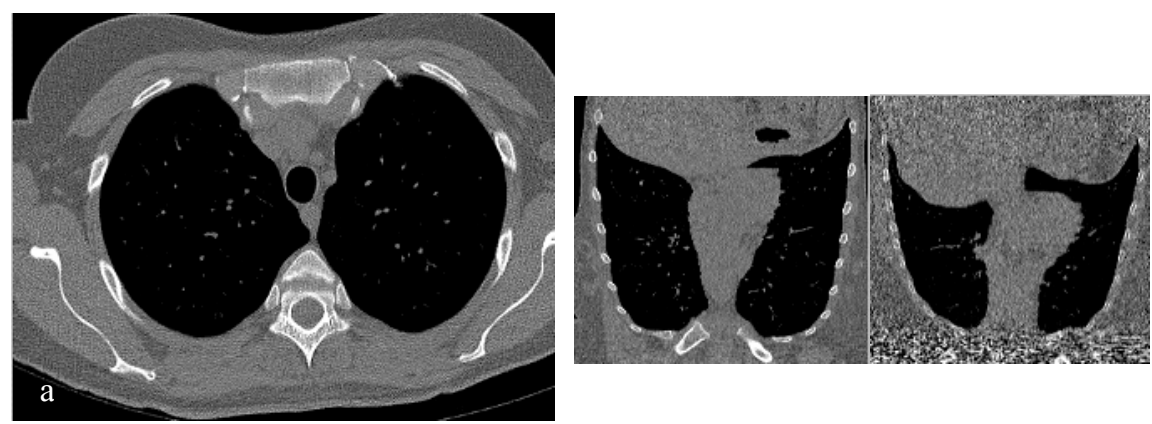

Fig. 1. (a) An axial slice image from a high dose chest CT volume data. The ellipsoid-like bright areas are the intersections of some of the individual ribs with this axial plane. Bottom left (b) and (c) Sagittal images of two volume data from high and low dose scans. Note the difference in noise level.

In the system viewpoint, the rib structures have high value in the reference and registration of other anatomies and pathologies.[3] The ribs have valuable properties common to bone tissues--rigid and stable in shape, and map to prominent intensities in CT data. Further, the rib structures enclose the complete chest area and part of lower abdomen, and are relatively less affected by lung surgery. Most importantly, they are highly ordered and symmetrical, and we observed that each pair of ribs roughly forms a plane-slanted plane that makes a significant angle with the axial plane. Because of these, the rib feature group can be used for reliable registration and reference. Certainly, to make full use of the structural advantage of the ribs, they should be extracted and labeled individually.

To our knowledge, there is little work in the 3D extraction of individual rib structures for the purpose of labeling or using them as features. In 3D volume data, efforts were spent on eliminating the ribs and other connected bone structures from the volume data by region-based approaches. [4]-[6] In the result, the ribs, spines and sternums were connected as one region. Moreover, the methods were semi-automaticrequires initial user input. In $2 \mathrm{D} x$-ray images, works existed on extraction of ribs, by fitting curves [7][8] or using pixel-based statistical classification method [9].

Our algorithm uses a tracing based approach. It is designed to extract individual rib centerlines and boundaries reliably in 3D volume data. At first thought on this segmentation problem, one would easily think of a region grow approach based on intensity values. First, the separation of spines and sternum from the grown region of bones is itself a challenging problem. Second, due to the hollowness of ribs, region-based method followed by skeletonization [8][9] will have difficulties in obtaining reliable centerlines that are important for registration. Finally, it is hard to obtain good result using an intensity based approach. Although the vast majority of bone voxels have higher intensities $(>1200)$ than surrounding tissues (700-1100), the lowest bone intensity and highest tissue intensity levels are quite close. With noise and partial volume 
effect, this narrow buffer zone is frequently crossed, and therefore no clear-cut intensity threshold exists between bone and other tissues.

The general principle of tracing has been widely explored, mostly in $2 \mathrm{~d}$, some in $3 \mathrm{~d}$ situations.[11] The difficulty in the rib tracing includes the following. First, it is a $3 \mathrm{~d}$ problem, with intrinsic issues to be handled. Second, the noise in low dose CT data can be high, leading to obscure and broken rib boundaries. The different noise levels can be seen from the pictures in Fig. 1(b) and (c). Third, the rib shapes vary greatly, making it hard to model. Fourth, the adjacent other bone structures may digress the tracing paths.

\section{Methods}

\subsection{Model, Assumptions, and Basic Methods}

An individual rib can be modeled as a tube-like structure, which is elongated, curved and internally hollow, as shown in Fig. 2(a). The cross-sections of a rib vary both in shape and size.
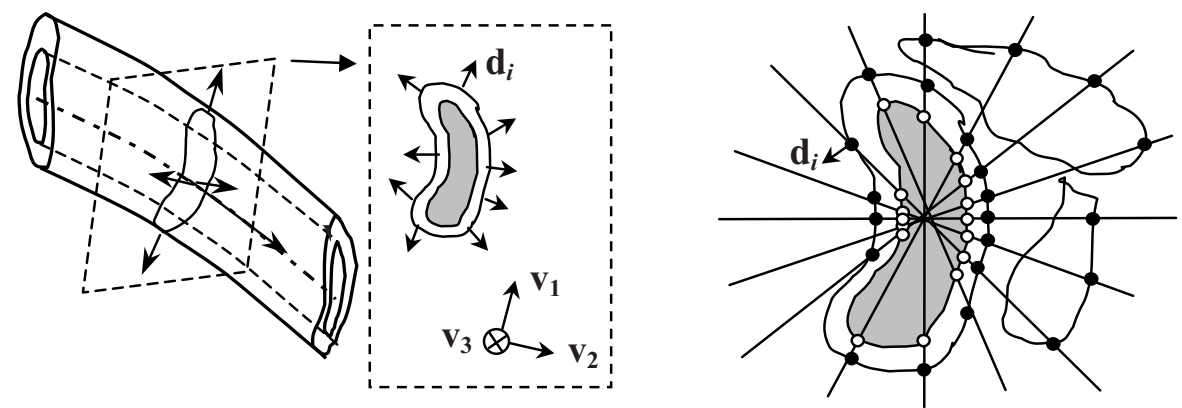

Fig. 2 (a) The geometric model of the rib structure. We assume the normals of the outer surface are perpendicular to the centerline direction (noted as $\mathbf{v}_{3}$ ) everywhere. At each tracing step, contour is extracted in the cross-section plane with normal $\mathbf{v}_{3}$. (b) At each step, all candidate edges are detected in each searching direction. The inner (white) edges are removed.

In our model, we assume that the outer surface is smooth everywhere, and more importantly, the surface normals $\mathbf{d}_{i}, i=1 \ldots n$ are roughly perpendicular to the centerline direction. This leads to our basic method of computing the tracing direction given the surface normals of detected edge points. Suppose the unit gradient vectors of $n$ edge points on a local cross-section represent the local surface normals, and we compute a covariance matrix of all unit vectors $\mathbf{d}_{i}$,

$$
\mathbf{C}=\sum_{i=1}^{n}\left(\mathbf{d}_{i}-\overline{\mathbf{d}}\right)^{t}\left(\mathbf{d}_{i}-\overline{\mathbf{d}}\right),
$$


where $\overline{\mathbf{d}}$ is average of all edge gradients. We compute the eigenvalues $\lambda_{1} \geq \lambda_{2} \geq \lambda_{3}$, with corresponding eigenvectors $\mathbf{v}_{1}, \mathbf{v}_{2}$, and $\mathbf{v}_{3}$. Since $\mathbf{v}_{3}$ is the new basis direction where the gradient vectors vary the least, it is perpendicular to the 2D subspace of the unit vectors. Therefore we take this vector as the centerline direction estimated from the gradient of local edges. The recursive tracing can then be described as

$$
\mathbf{p}^{(i+1)}=\mathbf{p}^{(i)}+\alpha \mathbf{v}_{3}
$$

in which the current centerline point $\mathbf{p}^{(i+1)}$ is determined from the previous centerline point $\mathbf{p}^{(\mathrm{i})}$, and $\alpha$ is the step size.

At each step, we define a cross-section plane whose normal is the previous centerline direction. As shown in Fig. 2(a), the intersection points of this plane and the rib outer surface form a rib contour, which are extracted using $3 \mathrm{~d}$ edge detection. . However, we do not perform edge detection on each pixel on the plane. Rather, as shown in Fig. 2(b), we extend 20 equal spaced search directions from the initial point $\mathbf{p}^{(\mathrm{i})}$ to look for strong edges. In many cases, more than one edge points are detected along each search direction.

\subsection{Seed Point Detection}

To obtain one seed point for each rib, we first select a sagittal plane close to the center of the chest. This plane makes intersections with all of the ribs, but does not contain any point from the spine or sternum. On this sagittal image, the intersections of the ribs are small ellipses lining up uniformly around the border of the lungs, see example images shown in Fig. 1(b) and (c).

A bone threshold is applied to binarize the image, and label the foreground regions. Size constraints are applied to rule out most of the false regions, and the centroids of the remaining regions are recorded as seed candidates. The contours of the lung boundaries are also extracted. Valid seed candidates are required to be close enough to the outer lung boundary, and uniformly distributed along the boundary.

Sometimes, due to partial volume effect and noises, the cross-section of a rib may split into more than one region. Hence, we merge the seed points that are very close to each other.

Using the above rules, all seed points are detected reliably from data of various noise levels. Depending on the range of the CT scan, we obtain 8-10 pairs of seed points. Starting at each seed point, we trace in both directions, and merge the two partial tracing results.

\subsection{Extraction of Rib Contour in Cross-Section Plane}

Instead of using a regular 3D edge operator to detect edges, we adapted the edge detector from the work of M. Brejl et al. [12] It is based on the fitting of the volume data in a small 3D voxel neighborhood by a polynomial. The resulting gradient computation yields accurate results, and has good performance at high noise levels. The algo- 
rithm can be implemented as a mask convolution with the mask size corresponding to the size of the voxel neighborhood within which the polynomial is fitted. We chose the mask size as $5 \times 5 \times 5$, which proves to be the best compromise between accuracy and speed. The masks can be described as a function of position $(x, y, z)$ :

$$
\begin{aligned}
& M_{x}(x, y, z)=\left(\mathrm{a}+b x^{2}-\mathrm{c} y^{2}-\mathrm{c} z^{2}\right) x \\
& M_{y}(x, y, z)=\left(\mathrm{a}-c x^{2}+b y^{2}-\mathrm{c} z^{2}\right) y \\
& M_{z}(x, y, z)=\left(\mathrm{a}-c x^{2}-\mathrm{c} y^{2}+b z^{2}\right) z
\end{aligned}
$$

where $\mathrm{a}=0.00214212, \mathrm{~b}=0.0016668, \mathrm{c}=0.000952378, x, y, z \in\{-2,-1,0,1,2\}$.

The above $3 \mathrm{D}$ masks are placed only around the points on the 20 search directions on the $2 \mathrm{D}$ cross-section plane whose normal is the previous tracing direction (Fig. 2). Edges are detected by convolution and non-maxima suppression along each search direction. Rib edge candidates are those edges that have high enough gradient magnitudes and with their gradient directions pointing out of the outer surface. Also, there must be enough high intensity bone voxels within their immediate neighborhood.

On each of the 20 search directions, there will be multiple edge candidates, only one of which is true. Some of the false edges are unfiltered inner edges and noise edges, but often they belong to nearby non-rib bone structures. A false edge can have a larger gradient magnitude than that of the true edge along a search direction.

To obtain the best contour, we use dynamic programming [13] to jointly estimate the best rib edge on each direction, taking into account both the shape and size of the rib contour.

From our model, the rib outer surface is smooth, and hence should result in compact and smooth contours in the cross-section plane. The energy function therefore contains terms measuring the length and total curvature of the contour. It is defined as

$$
E=\sum_{i}\left(E_{d}(i)+\alpha E_{c}(i)\right)
$$

where $E_{d}(i)$ is the distance between the two selected candidates on the $i^{\text {th }}$ and $i+1^{\text {th }}$ search direction, $E_{c}(i)$ is the correlation between two tangential vectors of these two points, and $\alpha$ is a weight factor.

With the defined energy function, we regard each search direction as a stage, and each edge candidate on that search direction as a state. The true rib edges are determined by minimizing the total energy. The path associated with the minimum energy is selected as the optimal path. The states, or edge candidates on the path are recorded the final rib edge points.

The centroid of the final edges in this cross-section is then computed as the rib centerline point. The next tracing direction is also updated. 


\subsection{Trace Validation, Correction, and Termination}

The rib structure signals, although prominent in CT data, can easily be deteriorated by noise, surrounding structures, and partial volume effect. Our assumptions and model do not hold for all points, when the rib shape is irregular. Due to these complications, the tracing validation at each step and the stopping criterions require careful design. They should be just lenient enough for a good trace to continue but terminate a trace when appropriate.

We record the tracing directions of the previous 10 steps, and require that the current direction be close enough to the average of these directions. We also require that the cross-section contour extracted at each step is associated with a high energy. A more basic rule requires that there should be valid edges in most of the search directions. In the paths from the initial center point to the rib edges there should be mostly high intensity voxels.

Failure to meet the above criterions usually indicates that the trace has entered the posterior or anterior terminals. However, a single event of failure will not terminate the trace. In such a case, we retrospectively make adjustments of the initial center location and the tracing direction estimated from the last step. The initial center location is allowed to move in the close neighborhood, and the initial tracing direction can be adjusted by adding small deviations. For each of these adjustments, we carry the tracing procedure once more to see it the stopping criterions are met.

If the retrospective test fails, then algorithm will decide that the trace should be terminated.
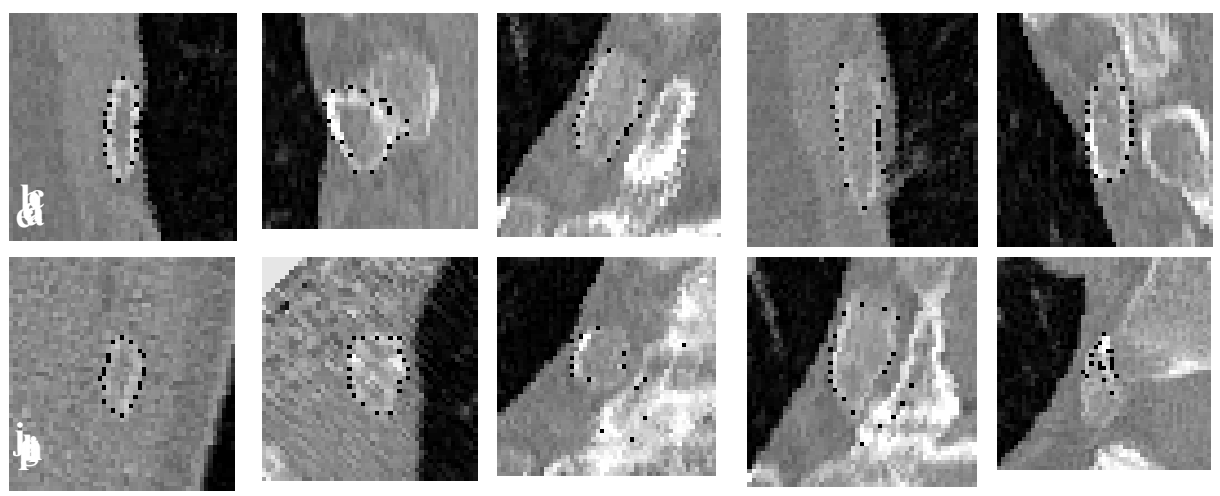

Fig. 3. Example cross-section contours. The black dots mark the contour edges determined by dynamic programming. (a)-(g): success cases $(\mathrm{h}-\mathrm{j})$ : failure cases with energy values higher than threshold.

\section{Results}

We test the algorithm on around 40 multi-slice chest CT data sets. In our experiments, the data sets are super-sampled into isotropic data. The size of the slice image is $512 \times 512$, and each isotropic volume data set contains about 400 500 slices. The 
resolution is in the range of $0.5 \sim 0.8 \mathrm{~mm}$ in each direction. Among these data sets, around half of them are from low-dose screening CT scans, and the rest is from highdose diagnostic scans.
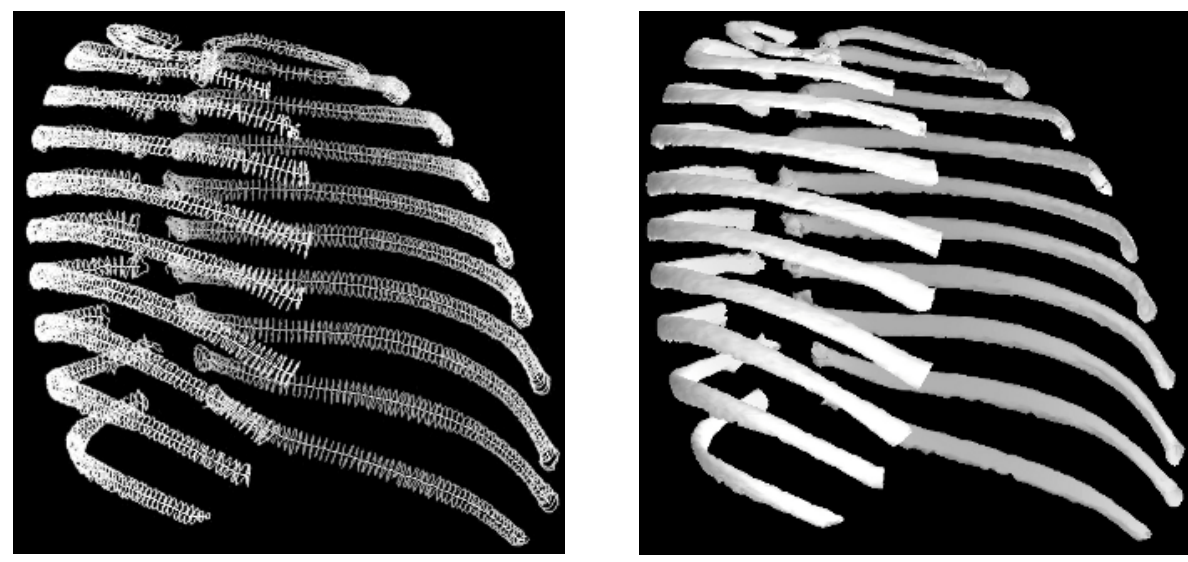

Fig. 4. An example result. (a) Extracted centerlines and contours that reflect the local shape. (b) Shaded surface display of the outer surface constructed from contours.

Shown in Fig. 3 are some example cross-section contours extracted during tracing. From pictures (a)-(g), we can see that the algorithm reliably extracts the contour edges despite noise and surrounding high intensity bone structures. Pictures (h)-(i) show cases where the energy function yields a high value, indicating a bad case. Some failure cases indicate that tracing has reached the spine terminals, and should be terminated; some are tolerated by the use of retrospective strategy. Generally, the edge detectors and dynamic programming techniques work well on convex and compact cross-section contours, but may have problem when the contours is very concave and even degenerate into a line-like structure.

The algorithm reliably extracts the centerlines, contours and outer surface of the rib structures, despite the various noise levels. Shown in Fig. 4 (a) and (b) is an example result. The surface in (b) is constructed with standard triangulation algorithms. The contours of the ribs reflect the local rib shapes, and the complete set of contours is an accurate description of the rib outer surface.

On a $2.8 \mathrm{GHz}$ Dual Processor Pentium IV PC, the extraction of the complete set of rib structures takes about 10 seconds.

\section{Conclusions}

We have developed a fast and robust algorithm for extraction of individual rib structures from CT volume data. The algorithm uses mainly edges, but intensity information is also taken into account. Statistical analysis and dynamic programming techniques are combined to achieve good result despite high noise level and the distur- 
bance of surrounding structures. Retrospective strategy with historical information is applied to help jumping through unfavorable cases.

The tracing algorithm has the advantage of extracting both the centerlines and contours at the same time. The 20 edge voxels at each step represent a sampling of local shape, forming the contour of the cross-section. All cross-section contours along the rib form the rib outer surface. These extracted features can be used for registration, visualization and other purposes.

Finally, the extracted ribs are ordered and labeled according to their relative spatial relationships and length ratios.

\section{References}

1. J. Qian, L. Fan, C. L. Novak, G. Wei, H. Shen, B. Odry G. Kohl D. P. Naidich, J. P. Ko, A. N. Rubinowitz, G. McGuinnes, "ICAD-Lung: An Interactive Computer Aided Diagnosis System for Lung Nodule Identification and Characterization" the Matrix Presentation at the European Congress of Radiology (ECR2002), Vienna, March, 2002.

2. H. Shen, L. Fan, J. Qian, B. L. Odry, C. L. Novak, and D.P. Naidich, Real-Time Correspondence Between Lung Nodules in Follow-Up Multi-Slice High Resolution CT Studies, the 2002 Conference of Radiological Society of North America, November, 2002.

3. H. Shen, and M. Shao, A Thoracic Cage Coordinate System for Recording Pathologies in Lung CT Volume Data, Proceedings of Nuclear Medicine Symposium and Medical Imaging Conference, Portland, OR, 2003.

4. D. Kim, H. Kim, and H. Kang, Object-tracking segmentation method: vertebra and rib segmentation in CT images, Proceedings of SPIE, vol.4684, pt.1-3, p.1662-71, 2002.

5. F. Vogelsang, F. Weiler, J. Dahmen, M. Kilbinger, B. Wein, and R. Gunther, Detection and compensation of rib structures in chest radiographs for diagnose assistance, Proceedings of SPIE, vol.3338, p.774-785, 1998.

6. G. Bohm, C.J.Knoll, M.L. Alcaniz-Raya, and S.E.Albalat, Three-dimensional segmentation of bone structures in CT images, Proceedings of Medical Imaging on Image Processing, pp 277-286, 1999.

7. De. Souza P, Automatic rib detection in chest radiographs, Computer Vision Graphics and Image Processing, vol.23, no.2, p.129-61, Aug. 1983.

8. F.Volgelsang, F. Weiler, J. Dahmen, M.Kilbinger, B.Wein, and R.W.Gunther, Detection and compensation of rib structures in chest radiographs for diagnostic assistance, Proceedings of the SPIE, vol.3338, pt.1-2, p.774-85, 1998.

9. M. Loog, B. van Ginneken, M.A. Viergever, Segmenting the Posterior Ribs in Chest Radiographs by Iterated Contextual Pixel Classification, Proceedings of SPIE, San Diego, CA, US, 2003.

10. P.Dimitrov, J.N.Damon, and K.Siddiqi, Flux invariants for shape, CVPR 2003: Computer Vision and Pattern Recognition Conference, vol.1, Madison, WI, USA, 18-20 June 2003.

11. A. Can, H. Shen, J.N.Turner, H.L. Tanenbaum, and B. Roysam, "Rapid Automated Tracing and Feature Extraction from Retinal Fundus Images Using Direct Exploratory Algorithms", IEEE Transactions on Information Technology in Biomedicine, vol. 3, No.2, pp. 125-138, June 1999.

12. M. Brejl and M. Sonka, Directional 3D edge detection in anisotropic data: detector design and performance assessment, Computer Vision and Image Understanding, vol.77, no.2, p.84-110, Feb. 2000.

13. E.V.Denardo, Models and Applications, Dover Publications, May, 2003. 